\title{
A Short Proof of Commutator Estimates
}

\section{Piero D'Ancona}

Journal of Fourier Analysis and Applications

ISSN 1069-5869

J Fourier Anal Appl

DOI 10.1007/s00041-018-9612-8

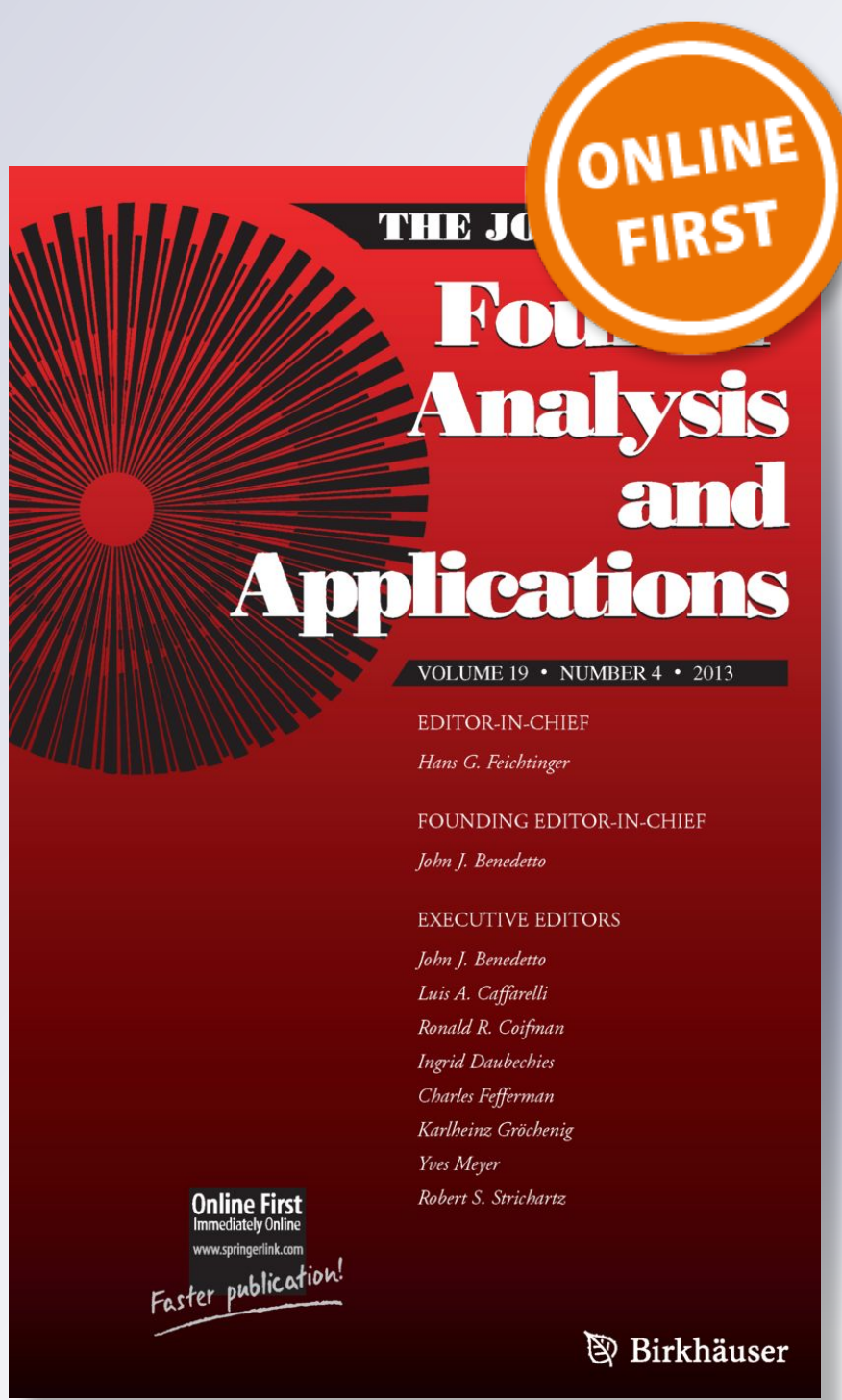

Springer 
Your article is protected by copyright and all rights are held exclusively by Springer Science+Business Media, LLC, part of Springer Nature. This e-offprint is for personal use only and shall not be self-archived in electronic repositories. If you wish to selfarchive your article, please use the accepted manuscript version for posting on your own website. You may further deposit the accepted manuscript version in any repository, provided it is only made publicly available 12 months after official publication or later and provided acknowledgement is given to the original source of publication and a link is inserted to the published article on Springer's website. The link must be accompanied by the following text: "The final publication is available at link.springer.com". 


\title{
A Short Proof of Commutator Estimates
}

\author{
Piero D'Ancona ${ }^{1}$
}

\begin{abstract}
The goal of this note is to give, at least for a restricted range of indices, a short proof of homogeneous commutator estimates for fractional derivatives of a product, using classical tools. Both $L^{p}$ and weighted $L^{p}$ estimates can be proved by the same argument. When the space dimension is 1 , we obtain some new estimates in the unexplored range $1 / 3<r \leq 1 / 2$.
\end{abstract}

Keywords Commutator estimates - Kato-Ponce estimates · Littlewwod square function · Muckenhoupt weights

\section{Introduction}

The homogeneous product estimate, also called fractional Leibniz rule, states that

$$
\left\|D^{S}(u v)\right\|_{L^{r}\left(\mathbb{R}^{n}\right)} \lesssim\left\|D^{S} u\right\|_{L^{p_{1}}}\|v\|_{L^{p_{2}}}+\|u\|_{L^{q_{1}}}\left\|D^{S} v\right\|_{L^{q_{2}}}
$$

for $u, v \in \mathscr{S}\left(\mathbb{R}^{n}\right)$, where $D^{s}=(-\Delta)^{s / 2}$.

Communicated by Fulvio Ricci.

$\triangle \quad$ Piero D’Ancona

dancona@mat.uniroma1.it

1 Dipartimento di Matematica, Sapienza Università di Roma, Piazzale A. Moro 2, 00185 Rome, Italy 
The conditions on the indices are

$$
\begin{aligned}
\frac{1}{r} & =\frac{1}{p_{1}}+\frac{1}{p_{2}} \\
& =\frac{1}{q_{1}}+\frac{1}{q_{2}}, \quad p_{j}, q_{j} \in(1, \infty], \quad s>\max \left(0, \frac{n}{r}-n\right) \text { or } s \in 2 \mathbb{Z}^{+} .
\end{aligned}
$$

When $r<1$ it is possible to take one of the indices $p_{j}$ (and $q_{i}$ ) equal to 1 , provided the $L^{r}$ quasinorm at the left is replaced by a $L^{r, \infty}$ seminorm (see [12,20] for the range $1<r<\infty$, Ref. [10] for the extension to values $r>1 / 2$, and [4] for the endpoint $r=p_{j}=q_{j}=\infty$ ). The proof relies on the Coifman-Meyer theory for bilinear multipliers [6] i.e. on paradifferential methods. Note that for integer values of $s$ the classical Gagliardo-Nirenberg estimates are sufficient to prove (1.1). An analogous estimate holds for the non homogeneous case where $D^{s}$ is replaced by $J^{s}:=(1-\Delta)^{s / 2}$.

Several variants and improvements of (1.1) are known. Indeed, the original result of Kato and Ponce is the following commutator estimate for $s>0$ :

$$
\left\|J^{s}(u v)-u J^{s} v\right\|_{L^{r}} \lesssim\left\|J^{s} u\right\|_{L^{p_{1}}}\|v\|_{L^{p_{2}}}+\|\partial u\|_{L^{q_{1}}}\left\|J^{s-1} v\right\|_{L^{q_{2}}} .
$$

An even stronger statement is due to Kenig et al. [13]:

$$
\left\|D^{S}(u v)-u D^{S} v-v D^{S} u\right\|_{L^{r}} \lesssim\left\|D^{S_{1}} u\right\|_{L^{p_{1}}}\left\|D^{S_{2}} v\right\|_{L^{p_{2}}}
$$

provided $s=s_{1}+s_{2}$ with $s, s_{j} \in(0,1)$ and $\frac{1}{r}=\frac{1}{p_{1}}+\frac{1}{p_{2}}$ with $r, p_{1}, p_{2} \in(1, \infty)$. Here the additional restrictions on $s, s_{j}$, are natural, but higher order versions of (1.4) have been obtained by Li [16] (see also [9]). The paper [16] gives a comprehensive view of the state of the art in this genre of inequalities, and in particular extends (1.4) to the range $1 / 2<r<1$. See also [14] for an alternative general approach to commutator estimates, based on the characterizations of functional spaces given in [5].

We also mention that product and Kato-Ponce commutator estimates in weighted $L^{p}$ spaces, with Muckenhoupt weights, have been recently proved by Cruz-Uribe and Naibo in [7], for the full range of indices (1.2) with the exception of the endpoint case $r=\infty$.

Our main purpose here is to give a very simple proof of the sharper estimate (1.4), relying entirely on classical tools of harmonic analysis. The main drawback is that in most cases the proof does not cover the full range of indices (1.2). However, in the allowed range of indices, the method is efficient, and indeed one recovers both unweighted and weighted estimates with essentially the same argument.

The main result of the paper is the following:

Theorem 1.1 Let $n \geq 1$. Assume $s, s_{1}, s_{2}$ and $r, p_{1}, p_{2}$ satisfy

$$
s=s_{1}+s_{2} \in(0,2), \quad s_{j} \in(0,1), \quad \frac{1}{r}=\frac{1}{p_{1}}+\frac{1}{p_{2}}, \quad \frac{2 n}{n+2 s_{j}}<p_{j}<\infty .
$$


Then for all $u, v \in \mathscr{S}\left(\mathbb{R}^{n}\right)$ we have

$$
\left\|D^{S}(u v)-u D^{S} v-v D^{S} u\right\|_{L^{r}} \lesssim\left\|D^{S_{1}} u\right\|_{L^{p_{1}}}\left\|D^{S_{2}} v\right\|_{L^{p_{2}}}
$$

Moreover, if we define

$$
q_{j}=p_{j}\left(\frac{1}{2}+\frac{s_{j}}{n}\right) \quad \text { if } n \geq 2, \quad q_{j}=\min \left\{p_{j}, p_{j}\left(\frac{1}{2}+s_{j}\right)\right\} \quad \text { ifn }=1,
$$

and we assume in addition $p_{1}, p_{2}>1$ when $n=1$, then for any weigths $w_{j} \in A_{q_{j}}$ we have

$$
\left\|D^{S}(u v)-u D^{S} v-v D^{S} u\right\|_{L^{r}\left(w_{1}^{r / p_{1}} w_{2}^{r / p_{2}} d x\right)} \lesssim\left\|D^{S_{1}} u\right\|_{L^{p_{1}}\left(w_{1} d x\right)}\left\|D^{S_{2}} v\right\|_{L^{p_{2}}\left(w_{2} d x\right)}
$$

We briefly discuss the result.

- The indices $r, p_{j}$ are in the ranges (recall that $s \in(0,2)$ and $s_{j} \in(0,1)$ )

$$
\frac{n}{n+s}<r<\infty, \quad \frac{2 n}{n+2 s_{j}}<p_{j}<\infty
$$

For $n \geq 2$ this is a strict subset of the known set given by $r \in\left(\frac{1}{2}, \infty\right], p_{j} \in(1, \infty]$. However, when $n=1$, the ranges are

$$
\frac{1}{1+s}<r<\infty \quad \frac{2}{1+2 s_{j}}<p_{j}<\infty
$$

so that $r$ can be arbitrarily close to $\frac{1}{3}$ and $p_{j}$ to $\frac{2}{3}$.

In particular, this shows that the range of indices in [10] is not sharp and can further be extended. This is likely due to the fact that paradifferential techniques do not adapt well to the range $p<1$. The result suggests that also in dimension $n \geq 2$ the usual range of indices may be extended below $1 / 2$.

- The 1-dimensional estimate can be applied also to functions of several variables, in the form (here $\left|\partial_{j}\right|^{s} u=\mathcal{F}^{-1}\left(\left|\xi_{j}\right|^{s} \widehat{u}\right)$ )

$$
\left\|\left|\partial_{j}\right|^{s}(u v)-u\left|\partial_{j}\right|^{s} v-v\left|\partial_{j}\right|^{s} u\right\|_{L^{r}} \lesssim\left\|\left|\partial_{j}\right|^{s_{1}} u\right\|_{L^{p_{1}}}\left\|\left|\partial_{j}\right|^{s_{2}} v\right\|_{L^{p_{2}}}
$$

for $j=1, \ldots, n$, and hence also multi-parameter estimates in the sense of $[18,19]$ can be deduced.

- One can deduce from (1.5) a fractional Leibniz rule via interpolation. For instance, in the one dimensional case, from (1.5) one has

$$
\left\|D^{S}(u v)\right\|_{L^{r}} \lesssim\left\|v D^{s} u\right\|_{L^{r}}+\left\|u D^{s} v\right\|_{L^{r}}+\left\|D^{s_{1}} u\right\|_{L^{r_{1}}}\left\|D^{s_{2}} v\right\|_{L^{r_{2}}} .
$$

To the first two terms one can apply Hölder's inequality. To the third term one applies a standard interpolation inequality

$$
\left\|D^{S_{1}} u\right\|_{L^{r_{1}}} \lesssim\left\|D^{S} u\right\|_{L^{p_{1}}}^{\theta}\|u\|_{L^{p_{3}}}^{1-\theta}
$$


(which follows from the complex interpolation formula $\dot{H}_{r_{1}}^{s_{1}}=\left[\dot{H}_{p_{1}}^{s}, L^{p_{2}}\right]_{\theta}$ with $s_{1}=(1-\theta) s+\theta \cdot 0$ and $\left.r_{1}^{-1}=(1-\theta) p_{1}^{-1}+\theta p_{2}^{-1}\right)$, and a similar one for $v$. Then by Cauchy-Schwartz one obtains (1.1).

- We relax the restriction $s<1$ in estimate (1.4) to $s<2$; note that this result is (marginally) sharper than the corresponding estimates in [16].

- The weighted estimates (1.6) are new; note however that in [7] weighted versions of the product estimates (1.1) and of the Kato-Ponce estimates (1.3) were proved, with conditions on the weights similar to ours.

- When $n=1$ and $s>1 / 2$, applying a result in [15], we get an explicit and sharp bound of the constant in (1.6), as a function of the Muckenhoupt norms of the weights (see Remark 2.6).

The proof is remarkably short and is based on the explicit representation

$$
\begin{aligned}
D^{s}(u v)-u D^{s} v-v D^{s} u & =c \int \frac{[u(x+y)-u(x)][v(x+y)-v(x)]}{|y|^{n+s}} d y, \\
0<s<2 & \text { e }
\end{aligned}
$$

for a suitable $c=c(n, s)$. From this we deduce the following pointwise bound

$$
\left|D^{s}(u v)-u D^{s} v-v D^{s} u\right| \lesssim g_{\lambda_{1}}^{*}\left(D^{s_{1}} u\right)(x) \cdot g_{\lambda_{2}}^{*}\left(D^{s_{2}} v\right)(x), \quad s_{1}+s_{2}=s
$$

in terms of the Littlewood nontangential square function $g_{\lambda}^{*}$. In this way classical $L^{p}$ and weighted $L^{p}$ bounds for $g_{\lambda}^{*}$ can be applied. The limitations on the set of indices are unavoidable due to well known counterexamples for the square functions (see [8]); it should be possible to obtain a more complete result by analyzing directly the Dirichlet form

$$
T_{s}(u, v)=\int \frac{[u(x+y)-u(x)][v(x+y)-v(x)]}{|y|^{n+s}} d y .
$$

Remark 1.2 Note that, thanks to the characterization of homogeneous Besov norms

$$
\|u\|_{\dot{B}_{p, q}^{s}}=\left\|\frac{u(x+y)-u(x)}{|y|^{s+n / q}}\right\|_{L_{y}^{q} L_{x}^{p}}, \quad 0<s<1, \quad p, q \in[1, \infty]
$$

a Besov version of the Kenig-Ponce-Vega estimates (1.4) is almost trivial to prove. Indeed, applying Hölder's inequality to (1.7) first in $x$ then in $y$, we get

$$
\left\|D^{s}(u v)-u D^{s} v-v D^{s} u\right\|_{L^{r}} \lesssim\|u\|_{\dot{B}_{p_{1}, q_{1}}^{s_{1}}}\|v\|_{\dot{B}_{p_{2}, q_{2}}^{s_{2}}}
$$

provided $s \in(0,2), s_{j} \in(0,1)$ and $r, p_{j}, q_{j} \in[1, \infty]$ satisfy

$$
s=s_{1}+s_{2}, \quad \frac{1}{r}=\frac{1}{p_{1}}+\frac{1}{p_{2}}, \quad 1=\frac{1}{q_{1}}+\frac{1}{q_{2}} .
$$


Remark 1.3 Besides commutator estimates, a similar approach can be used to study the fractional p-Laplacian

$$
(-\Delta)_{p}^{s} u=c(n, s, p) \int_{\mathbb{R}^{n}} \frac{|u(x)-u(y)|^{p-2}[u(x)-u(y)]}{|x-y|^{n+s p}} d y
$$

and more general Dirichlet forms like

$$
\mathcal{E}(u, v)=\iint(u(x)-u(y))(v(x)-v(y)) \frac{A(x, y)}{|x-y|^{n+a}} d y d x \quad a \in(0,2)
$$

with $\Lambda \geq A(x, y) \geq \Lambda^{-1}>0$, see e.g. $[3,11]$

\section{The Proofs}

We begin by recalling the explicit representation for fractional derivatives as a hypersingular integral, sometimes named after Aronszajn and Smith:

Lemma 2.1 [2] For all $u \in \mathscr{S}\left(\mathbb{R}^{n}\right), x \in \mathbb{R}^{n}$, and $0<s<2$ we have

$$
D^{s} u(x)=c(n, s) \cdot \lim _{\epsilon \downarrow 0} \int_{|x|>\epsilon} \frac{u(x+y)-u(x)}{|y|^{n+s}} d y .
$$

Proof Consider the identity

$$
\begin{aligned}
\Delta_{y} \frac{u(x+y)-u(x)}{|y|^{n+s-2}}= & \frac{\Delta_{y} u(x+y)}{|y|^{n+s-2}-2 c \nabla_{y}} \cdot\left\{y \frac{u(x+y)-u(x)}{|y|^{n+s}}\right\} \\
& -s c \frac{u(x+y)-u(x)}{|y|^{n+s}}
\end{aligned}
$$

where $c=n+s-2$. If we integrate over $\left\{y \in \mathbb{R}^{n}:|y|>\epsilon\right\}$ and let $\epsilon \downarrow 0$ we obtain

$$
\int \frac{\Delta_{y} u(x+y)}{|y|^{n+s-2}} d y=s c \cdot \lim _{\epsilon \downarrow 0} \int_{|x|>\epsilon} \frac{u(x+y)-u(x)}{|y|^{n+s}} d y .
$$

Since the first integral is precisely

$$
\int \frac{\Delta_{y} u(x+y)}{|y|^{n+s-2}} d y=|\cdot|^{-n-s+2} * \Delta u(x)=c^{\prime} D^{s-2} \Delta u=c^{\prime} D^{s} u
$$

for a suitable constant $c^{\prime}=c^{\prime}(n, s)$, the proof is concluded.

Thus $D^{s} u$ can be written as the principal value integral (2.1); note that in the range $0<s<1$ (and for smooth $u$ ) the integral is actually absolutely convergent. In the following we shall write simply

$$
\int \frac{u(x+y)-u(x)}{|y|^{n+s}} d y \text { instead of P.V. } \int \frac{u(x+y)-u(x)}{|y|^{n+s}} d y .
$$


Writing $u_{ \pm}=u(x \pm y), v_{ \pm}=v(x \pm y), u=u(x), v=v(x)$, one has the identity

$$
\left(u_{+} v_{+}-u v\right)-u\left(v_{+}-v\right)-\left(u_{+}-u\right) v=\left(u_{+}-u\right)\left(v_{+}-v\right),
$$

thus (2.1) implies the formula

$$
D^{s}(u v)-u D^{s} v-v D^{s} u=c(n, s) \cdot T_{s}(u, v), \quad 0<s<2
$$

where $T_{S}(u, v)$ is the bilinear form

$$
T_{S}(u, v)(x)=\int \frac{[u(x+y)-u(x)][v(x+y)-v(x)]}{|y|^{n+s}} d y, \quad 0<s<2 .
$$

Remark 2.2 It is possible to work exclusively with absolutely convergent integrals, using the equivalent representation

$$
D^{s} u(x)=c \int \frac{u(x+y)+u(x-y)-2 u(x)}{|y|^{n+s}} d y
$$

and the identity

$$
\begin{aligned}
& \left(u_{+} v_{+}+u_{-} v_{-}-2 u v\right)-u\left(v_{+}+v_{-}-2 v\right) \\
& \quad-\left(u_{+}+u_{-}-2 u\right) v=\left(u_{+}-u\right)\left(v_{+}-v\right)+\left(u-u_{-}\right)\left(v-v_{-}\right) .
\end{aligned}
$$

In order to estimate $T_{S}(u, v)$ we use the square fractional integral (see [21])

$$
\mathcal{D}_{\gamma}[u](x)=\left(\int \frac{|u(x+y)-u(x)|^{2}}{|y|^{n+2 \gamma}} d y\right)^{\frac{1}{2}}, \quad 0<\gamma<1 .
$$

By Cauchy-Schwartz one has the pointwise bound

$$
\left|T_{s}(u, v)\right| \leq \mathcal{D}_{s_{1}}[u] \mathcal{D}_{s_{2}}[v], \quad s=s_{1}+s_{2}, \quad s \in(0,2), s_{j} \in(0,1)
$$

and we are reduced to estimate the fractional integral $\mathcal{D}_{s}$. To this end, we shall use the Littlewood nontangential square function $g_{\lambda}^{*}(u)$ defined as follows $\left(\partial_{x, t}=\right.$ $\left.\left(\partial_{x_{1}}, \ldots, \partial_{x_{n}}, \partial_{t}\right)\right)$ :

$$
g_{\lambda}^{*}(u)(x)=\left[\int_{0}^{\infty} \int_{\mathbb{R}^{n}}\left(\frac{t}{t+|y|}\right)^{\lambda n} t^{1-n}\left|\partial_{t, x} U(x-y, t)\right|^{2} d y d t\right]^{\frac{1}{2}}
$$

where $U(x, t)$ is the harmonic extension of $u(x)$ in the upper half space $\mathbb{R}_{x, t}^{n+1}$ :

$$
U(x, t)=e^{-t D} u=\frac{\Gamma\left(\frac{n+1}{2}\right)}{\pi^{\frac{n+1}{2}}} \int_{\mathbb{R}^{n}} \frac{t u(x-y)}{\left(t^{2}+|y|^{2}\right)^{\frac{n+1}{2}}} d y, \quad x \in \mathbb{R}^{n}, t>0 .
$$

Now, the crucial step is the following pointwise estimate: 
Theorem 2.3 [21] Let $n \geq 1,0<s<1$ and $\lambda<1+\frac{2 s}{n}$. Then we have

$$
\mathcal{D}_{s}[u](x) \leq c(n, s) g_{\lambda}^{*}\left(D^{s} u\right)(x)
$$

with a constant independent of $u \in \mathscr{S}\left(\mathbb{R}^{n}\right)$ and $x \in \mathbb{R}^{n}$.

Proof The result is stated in [21] and a hint is given in [23]. For the sake of completeness, we include a proof in the Appendix of the paper.

Summing up, we have proved the following

Proposition 2.4 (Pointwise commutator estimate) Let $n \geq 1$ and

$$
s=s_{1}+s_{2} \in(0,2), \quad s_{j} \in(0,1), \quad \lambda_{j}<1+\frac{2 s_{j}}{n} .
$$

Then the following pointwise estimate holds, with a constant independent of $u, v \in$ $\mathscr{S}\left(\mathbb{R}^{n}\right)$ and $x \in \mathbb{R}^{n}$ :

$$
\left|D^{S}(u v)-u D^{s} v-v D^{s} u\right| \lesssim g_{\lambda_{1}}^{*}\left(D^{s_{1}} u\right)(x) \cdot g_{\lambda_{2}}^{*}\left(D^{s_{2}} v\right)(x)
$$

It remains to estimate the square functions at the right of (2.5). We recall a few well known properties of $g_{\lambda}^{*}$ :

Theorem 2.5 Let $n \geq 1, \lambda>1$. For any $u \in \mathscr{S}\left(\mathbb{R}^{n}\right), g_{\lambda}^{*}(u)$ satisfies the following estimates, with constants independent of $u$ :

(i) $\left\|g_{\lambda}^{*}(u)\right\|_{L^{p}} \lesssim\|u\|_{L^{p}}$ for $\lambda>\max \left\{1, \frac{2}{p}\right\}$ and $0<p<\infty$

(ii) $\left\|g_{\lambda}^{*}\right\|_{L^{p}(w d x)} \lesssim\|u\|_{L^{p}(w d x)}$ for $\lambda>\max \left\{1, \frac{2}{p}\right\}, 1<p<\infty$ and $w \in$ $A_{\min \left\{p, \frac{p \lambda}{2}\right\}}$.

Proof Estimate (i) is proved in [1,22,23]; see also [24] for a proof in the range $0<$ $p \leq 1$. Estimate (ii) is from [17] (Corollary at p. 110).

In the borderline case $\lambda=2 / p$ estimate (i) is valid with $L^{p, \infty}$ in place of $L^{p}$ at the left $[1,8]$. The corresponding weighted weak estimate is contained in [17]; moreover, estimate (ii) holds also for $p \leq 1$ provided the weighted $L^{p}$ norm at the left is replaced by a weighted Hardy space norm (see [17]). The sharp form of the constant in estimate (ii) is known if $\lambda>2,1<p<\infty$ :

$$
\left\|g_{\lambda}^{*}\right\|_{L^{p}(w d x)} \leq C(n, p, \lambda)[w]_{A_{p}}^{\max }\left\{\frac{1}{2}, \frac{1}{p-1}\right\}\|u\|_{L^{p}(w d x)}
$$

as proved in [15], but it is still unkown for $\lambda \leq 2$. Recall that $[w]_{A_{p}}$ for $1<p<\infty$ is the minimal $C$ such that the averages over any ball $B \subset \mathbb{R}^{n}$ satisfy

$$
f_{B} w \cdot\left(f_{B} w^{-\frac{1}{p-1}}\right)^{p-1} \leq C .
$$

Now it is a simple matter to prove the main result: 
Proof of Theorem 1.1 By Hölder's inequality and (2.5) we have

$$
\left\|D^{s}(u v)-u D^{s} v-v D^{s} u\right\|_{L^{r}} \lesssim\left\|g_{\lambda_{1}}^{*}\left(D^{s_{1}} u\right)\right\|_{L^{p_{1}}}\left\|g_{\lambda_{2}}^{*}\left(D^{s_{2}} v\right)\right\|_{L^{p_{2}}}
$$

for any $r, p_{1}, p_{2} \in(0, \infty]$ with $\frac{1}{r}=\frac{1}{p_{1}}+\frac{1}{p_{2}}$, any $s_{j} \in(0,1)$ and any $\lambda_{j}<1+\frac{2 s_{j}}{n}$. Applying Theorem 2.5(i) we get (1.5) provided we can pick $\lambda_{j}$ such that

$$
\max \left\{1, \frac{2}{p_{j}}\right\}<\lambda_{j}<1+\frac{2 s_{j}}{n}
$$

which is possible by the conditions on $p_{j}, s_{j}$.

The second estimate is proved in a similar way using Theorem 2.5(ii). We obtain the following condition on the weights:

$$
w_{j} \in A_{q_{j}}, \quad 1<q_{j}<\min \left\{p_{j}, p_{j}\left(\frac{1}{2}+\frac{s_{j}}{n}\right)\right\} .
$$

Thanks to the self improving property of Muckenhoupt classes (i.e., if $w \in A_{q}$ with $q>1$ then $w \in A_{q_{1}}$ for some $q_{1}<q$ ), we can relax the condition to

$$
w_{j} \in A_{q_{j}}, \quad 1<q_{j}=\min \left\{p_{j}, p_{j}\left(\frac{1}{2}+\frac{s_{j}}{n}\right)\right\} .
$$

In dimensions $n \geq 2$ we have always $s_{j} / n \leq 1 / 2$ and hence we obtain

$$
w_{j} \in A_{q_{j}}, \quad 1<q_{j}=p_{j}\left(\frac{1}{2}+\frac{s_{j}}{n}\right),
$$

while in dimension $n=1$ we have

$$
w_{j} \in A_{q_{j}}, \quad 1<q_{j}=\min \left\{p_{j}, p_{j}\left(\frac{1}{2}+s_{j}\right)\right\}
$$

and the proof is concluded.

Remark 2.6 In the case $n=1$ and $s_{1}, s_{2}>1 / 2$, the values of $\lambda_{1}, \lambda_{2}$ in the previous proof can be taken both $>2$ and then Lerner's result [15] gives the following explicit bound on the constant of (1.6): if $p_{1}, p_{2}>1$,

$$
C \leq c\left(n, a, s_{j}, r, p_{j}\right)\left[w_{1}\right]_{A_{p_{1}}}^{\max }\left\{\frac{1}{2}, \frac{1}{p_{1}-1}\right\}\left[w_{2}\right]_{A_{p_{2}}}^{\max }\left\{\frac{1}{2}, \frac{1}{p_{2}-1}\right\} .
$$

\section{Appendix A: Proof of Theorem 2.3}

It is sufficient to prove the inequality at $x=0$. Let $u \in \mathscr{S}\left(\mathbb{R}^{n}\right)$ and let $U(x, t)=e^{-t D} u$ be its harmonic extension on $\mathbb{R}_{+}^{n+1}$ for $t>0$. Following the hint in [23] V.6.12, we can estimate the difference $u(y)-u(0)$ with the integral of $\partial_{x, t} U(x, t)$ along any path contained in $\mathbb{R}_{+}^{n+1}$ joining the points $(0,0)$ and $(y, 0)$. We choose a path made by a vertical segment joining $(0,0)$ with $(0,|y|)$, followed by a horizontal segment joining 
$(0,|y|)$ with $(y,|y|)$, followed by a vertical segment joining $(y,|y|)$ with $(y, 0)$. We get

$$
|u(y)-u(0)| \leq \int_{0}^{|y|}(|\partial U(y, \lambda)|+|\partial U(0, \lambda)|+|\partial U(\lambda \widehat{y},|y|)|) d \lambda
$$

where $\hat{y}=y /|y|$. If we denote with $F(x, t)=e^{-t D} D^{s} u$ the harmonic extension of $D^{s} u$, we have the formula

$$
U(z, s)=\int_{0}^{\infty} F(z, t+\mu) \mu^{s-1} d \mu
$$

which implies

$$
\begin{aligned}
& |u(y)-u(0)| \leq \int_{0}^{|y|} \int_{\lambda}^{\infty}(|\partial F(y, \mu)| \\
& \quad+|\partial F(0, \mu)|+|\partial F(\lambda \widehat{y}, \mu+|y|-\lambda)|)(\mu-\lambda)^{s-1} d \mu d \lambda .
\end{aligned}
$$

We split the RHS in the sum of four pieces $I+I I+I I I+I V$ where

$$
\begin{aligned}
I & =\int_{0}^{|y|} \int_{\lambda}^{|y|}|\partial F(y, \mu)|(\mu-\lambda)^{s-1} d \mu d \lambda, \\
I I & =\int_{0}^{|y|} \int_{\lambda}^{|y|}|\partial F(0, \mu)|(\mu-\lambda)^{s-1} d \mu d \lambda, \\
I I I & =\int_{0}^{|y|} \int_{\lambda}^{|y|}|\partial F(\lambda \widehat{y}, \mu+|y|-\lambda)|(\mu-\lambda)^{s-1} d \mu d \lambda, \\
I V & =\int_{0}^{|y|} \int_{|y|}^{\infty}(|\partial F(y, \mu)|+|\partial F(0, \mu)|+|\partial F(\lambda \widehat{y}, \mu+|y|-\lambda)|)(\mu-\lambda)^{s-1} d \mu d \lambda .
\end{aligned}
$$

The term $I V$ can be estimated for any $A$ with

$$
I V \lesssim \sup _{|z| \leq|y| \leq \lambda} \lambda^{A}|\partial F(z, \lambda)| \cdot \int_{0}^{|y|} \int_{|y|}^{\infty} \mu^{-A}(\mu-\lambda)^{s-1} d \mu d \lambda
$$

The integral is finite if $s<A<1$ and we get

$$
I V \lesssim \sup _{|z| \leq|y| \leq \lambda} \lambda^{A}|y|^{1+s-A}|\partial F(z, \lambda)|
$$

Using the mean value property of the harmonic function $\partial F$, we get

$$
(I V)^{2} \lesssim \sup _{|z| \leq|y| \leq \lambda} \lambda^{2 A}|y|^{2+2 s-2 A} \lambda^{-n-1} \int_{D}|\partial F(\xi, \tau)|^{2} d \xi d \tau
$$

where

$$
D=\{(\xi, \tau):|\xi-z| \leq \lambda / 2,|\tau-\lambda| \leq \lambda / 2\}
$$


Now we note that

$$
D \subset\{(\xi, \tau) \in \Gamma, \tau \geq|y| / 2\}
$$

where $\Gamma$ is the cone of aperture 3

$$
\Gamma=\{(\xi, \tau):|\xi| \leq 3 \tau\}
$$

and moreover, if $(\xi, \tau) \in D$, we have $\tau \simeq \lambda$ and actually $\lambda / 2 \leq \tau \leq 3 \lambda / 2$. This gives

$$
(I V)^{2} \lesssim|y|^{2+2 s-2 A} \int_{\Gamma, \tau \geq|y| / 2}|\partial F(\xi, \tau)|^{2} \tau^{2 A-n-1} d \xi d \tau
$$

Now dividing by $|y|^{n+2 s}$ and integrating in $y$ we have, inverting the order of integration,

$$
\int \frac{(I V)^{2}}{|y|^{n+2 s}} d y \lesssim \int_{\Gamma}\left(\int_{|y| \leq 2 \tau}|y|^{2-n-2 A} d y\right)|\partial F(\xi, \tau)|^{2} \tau^{2 A-n-1} d \xi d \tau
$$

and finally

$$
\int \frac{(I V)^{2}}{|y|^{n+2 s}} d y \lesssim \int_{\Gamma}|\partial F(\xi, \tau)|^{2} \tau^{1-n} d \xi d \tau
$$

(In particular we see that the piece $I V$ is estimated by a Lusin area integral on a cone of fixed aperture).

The terms $I I I$ and $I I$ satisfy an estimate similar to $I V$. Indeed, in $I I I$ we have $\mu+$ $|y|-\lambda \geq|y| \geq \lambda=|\lambda \widehat{y}|$ so that

$$
I I I \lesssim \sup _{|z| \leq|y| \leq \lambda} \lambda^{A}|\partial F(z, \lambda)| \cdot \int_{0}^{|y|} \int_{\lambda}^{|y|}(|y|+\mu-\lambda)^{-A}(\mu-\lambda)^{s-1} d \mu d \lambda ;
$$

using $(|y|+\mu-\lambda)^{-A} \leq|y|^{-A}$ we have

$$
\int_{0}^{|y|} \int_{\lambda}^{|y|}(|y|+\mu-\lambda)^{-A}(\mu-\lambda)^{s-1} d \mu d \lambda \leq|y|^{-A} \cdot|y|^{1+s}
$$

so that

$$
I I I \lesssim \sup _{|z| \leq|y| \leq \lambda} \lambda^{A}|y|^{1+s-A}|\partial F(z, \lambda)|
$$

Proceeds as for $I V$ we obtain that $I I I$ satisfies (A.1). 
For the term $I I$ we have by Fubini and then by Cauchy-Schwartz, for $\epsilon \in(0,2 s)$,

$$
I I \simeq \int_{0}^{|y|}|\partial F(0, \mu)| \mu^{s} d \mu \lesssim\left(\int_{0}^{|y|}|\partial F(0, \mu)|^{2} \mu^{1+2 s-\epsilon} d \mu\right)^{1 / 2}|y|^{\epsilon / 2}
$$

which gives

$$
\begin{aligned}
\int \frac{(I I)^{2}}{|y|^{n+2 s}} d y & \lesssim \int|y|^{\epsilon-n-2 s} \int_{0}^{|y|}|\partial F(0, \mu)|^{2} \mu^{1+2 s-\epsilon} d \mu \\
& =\int_{0}^{\infty}|\partial F(0, \mu)|^{2} \mu^{1+2 s-\epsilon} \int_{|y| \geq \mu} \frac{d y}{|y|^{n+2 s-\epsilon}} d \mu
\end{aligned}
$$

so that

$$
\int \frac{(I I)^{2}}{|y|^{n+2 s}} d y \lesssim \int_{0}^{\infty}|\partial F(0, \mu)|^{2} \mu d \mu
$$

We now apply the mean value property:

$$
\int \frac{(I I)^{2}}{|y|^{n+2 s}} d y \lesssim \int_{0}^{\infty} \mu^{-n} \int_{|\xi| \leq \mu / 2,|\tau-\mu| \leq \mu / 2}|\partial F(\xi, \tau)|^{2} d \xi d \tau d \mu .
$$

Note that the domain of integration of the inner integral is contained in the cone $\Gamma$ defined above, and that $\mu \simeq \tau$; more precisely we have

$$
\frac{\mu}{2} \leq \tau \leq \frac{3 \mu}{2} \text { i.e. } \frac{2 \tau}{3} \leq \mu \leq 2 \tau
$$

This gives, after exchanging the order of integration,

$$
\int \frac{(I I)^{2}}{|y|^{n+2 s}} d y \lesssim \int_{\Gamma}|\partial F(\xi, \tau)|^{2} \tau^{-n} \int_{2 \tau / 3}^{2 \tau} d \mu d \xi d \tau
$$

and finally we get as in (A.1)

$$
\int \frac{(I I)^{2}}{|y|^{n+2 s}} d y \lesssim \int_{\Gamma}|\partial F(\xi, \tau)|^{2} \tau^{1-n} d \xi d \tau
$$

The remaining piece $I$ is the only one requiring cones of arbitrary aperture, and hence the function $g_{\lambda}^{*}$. Exchanging the order of integration and using Cauchy-Schwartz we get

$$
I=s^{-1} \int_{0}^{|y|}|\partial F(y, \mu)| \mu^{s} d \mu \lesssim\left(\int_{0}^{|y|}|\partial F(y, \mu)|^{2} \mu^{1+2 s-\epsilon} d \mu\right)^{1 / 2}|y|^{\epsilon / 2}
$$


for any $\epsilon \in(0,2 s)$. Thus we have

$$
\int \frac{(I)^{2}}{|y|^{n+2 s}} d y \lesssim \iint_{0}^{|y|}|\partial F(y, \mu)|^{2} \mu^{1+2 s-\epsilon} d \mu|y|^{\epsilon-n-2 s} d y
$$

that is to say

$$
\int \frac{(I)^{2}}{|y|^{n+2 s}} d y \lesssim \int_{|y| \geq \mu}|\partial F(y, \mu)|^{2} \frac{\mu^{1+2 s-\epsilon}}{|y|^{n+2 s-\epsilon}} d y d \mu
$$

Summing all the pieces, we have proved the estimate

$\int \frac{\left|u(x+y)-u_{y}\right|^{2}}{|y|^{n+2 s}} d y \lesssim \int_{\Gamma}|\partial F(\xi, \tau)|^{2} \tau^{1-n} d \xi d \tau+\int_{|y| \geq \mu}|\partial F(y, \mu)|^{2} \frac{\mu^{1+2 s-\epsilon}}{|y|^{n+2 s-\epsilon}} d y d \mu$.

The first term at the right is obviously bounded by $g_{\lambda}^{*}\left(D^{s} u\right)(0)$ for all $\lambda$, while it is easy to check that the second integral is bounded by $g_{\lambda}^{*}\left(D^{s} u\right)(0)$ provided $\lambda=1+\frac{2 s}{n}-\frac{\epsilon}{n}$. Since $\epsilon$ is arbitrarily small, the proof is concluded.

\section{References}

1. Aguilera, N., Segovia, C.: Weighted norm inequalities relating the $g_{\lambda}^{*}$ and the area functions. Studia Math. 61(3), 293-303 (1977)

2. Aronszajn, N., Smith, K.T.: Theory of Bessel potentials. I. Ann. Inst. Fourier (Grenoble) 11, 385-475 (1961)

3. Bass, R.F., Ren, H.: Meyers inequality and strong stability for stable-like operators. J. Funct. Anal. 265(1), 28-48 (2013)

4. Bourgain, J., Li, D.: On an endpoint Kato-Ponce inequality. Differ. Integral Equ. 27(11-12), 1037-1072 (2014)

5. Bui, H.Q., Candy, T.: A characterisation of the Besov-Lipschitz and Triebel-Lizorkin spaces using Poisson like kernels. Functional Analysis, Harmonic Analysis, and Image Processing: A Collection of Papers in Honor of Björn Jawerth. Contemporary Mathematics, vol. 693, pp. 109-141. American Mathematical Society, Providence (2017) arXiv:1502.06836

6. Coifman, R.R., Meyer, Y.: Nonlinear harmonic analysis, operator theory and P.D.E. In: Beijing Lectures in Harmonic Analysis (Beijing, 1984), Annals of Mathematical Studies, vol. 112, pp. 3-45. Princeton University Press, Princeton (1986)

7. Cruz-Uribe, D., Naibo, V.: Kato-Ponce inequalities on weighted and variable Lebesgue spaces. Differ. Integral Equ. 29(9-10), 801-836 (2016)

8. Fefferman, C.: Inequalities for strongly singular convolution operators. Acta Math. 124, 9-36 (1970)

9. Fujiwara, K., Georgiev, V., Ozawa, T.: Higher order fractional Leibniz rule. J. Fourier Anal. Appl. (in press)

10. Grafakos, L., Oh, S.: The Kato-Ponce inequality. Commun. Partial Differ. Equ. 39(6), 1128-1157 (2014)

11. Ishii, H., Nakamura, G.: A class of integral equations and approximation of $p$-Laplace equations. Calc. Var. Partial Differ. Equ. 37(3-4), 485-522 (2010)

12. Kato, T., Ponce, G.: Commutator estimates and the Euler and Navier-Stokes equations. Commun. Pure Appl. Math. 41(7), 891-907 (1988)

13. Kenig, C.E., Ponce, G., Vega, L.: Well-posedness and scattering results for the generalized Kortewegde Vries equation via the contraction principle. Commun. Pure Appl. Math. 46(4), 527-620 (1993)

14. Lenzmann, E., Schikorra, A.: Sharp commutator estimates via harmonic extensions. arXiv:1609.08547 (2016)

\section{Birkhäuser}


15. Lerner, A.K.: On sharp aperture-weighted estimates for square functions. J. Fourier Anal. Appl. 20(4), 784-800 (2014)

16. Li, D.: On Kato-Ponce and fractional Leibniz rule (2016). arXiv:1609.01780v1

17. Muckenhoupt, B., Wheeden, R.L.: Norm inequalities for the Littlewood-Paley function $g_{\lambda}^{*}$. Trans. Am. Math. Soc. 191, 95-111 (1974)

18. Muscalu, C., Pipher, J., Tao, T., Thiele, C.: Bi-parameter paraproducts. Acta Math. 193(2), 269-296 (2004)

19. Muscalu, C., Pipher, J., Tao, T., Thiele, C.: Multi-parameter paraproducts. Rev. Mat. Iberoam. 22(3), 963-976 (2006)

20. Ponce, G.: On the global well-posedness of the Benjamin-Ono equation. Differ. Integral Equ. 4(3), 527-542 (1991)

21. Stein, E.M.: The characterization of functions arising as potentials. Bull. Am. Math. Soc. 67, 102-104 (1961)

22. Stein, E.M.: On some funcions of Littlewood-Paley and Zygmund. Bull. Am. Math. Soc. 67, 99-101 (1961)

23. Stein, E.M.: Singular Integrals and Differentiability Properties of Functions. Princeton Mathematical Series, No. 30. Princeton University Press, Princeton (1970)

24. Torchinsky, A.: Real-Variable Methods in Harmonic Analysis. Pure and Applied Mathematics, vol. 123. Academic Press, Orlando (1986) 